\title{
Theranostics
}

Research Paper

2017; 7(1): 153-163. doi: 10.7150/thno.17085

\section{Mapping Sentinel Lymph Node Metastasis by Dual-probe Optical Imaging}

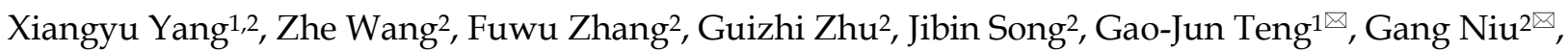 \\ Xiaoyuan Chen ${ }^{2}$ \\ 1. Jiangsu Key Laboratory of Molecular Imaging and Functional Imaging, Department of Radiology, Zhongda Hospital, Medical School of Southeast \\ University, Nanjing 210009, China; \\ 2. Laboratory of Molecular Imaging and Nanomedicine, National Institute of Biomedical Imaging and Bioengineering, National Institutes of Health (NIH), \\ Bethesda, MD 20892, USA. \\ $\triangle$ Corresponding authors: Gao-Jun Teng, 87 Dingjiaqiao Road, Nanjing, Jiangsu, China, 210009, Email: giteng@vip.sina.com Gang Niu, 35A Convent Drive Rm \\ GD959, Bethesda, MD 20892, Email: niug@mail.nih.gov Xiaoyuan Chen, 35A Convent Drive Rm GD937, Bethesda, MD 20892, Email: shawn.chen@nih.gov.
}

(0) Ivyspring International Publisher. Reproduction is permitted for personal, noncommercial use, provided that the article is in whole, unmodified, and properly cited. See http://ivyspring.com/terms for terms and conditions.

Received: 2016.08.02; Accepted: 2016.10.04; Published: 2017.01.01

\begin{abstract}
Purpose: Sentinel lymph node biopsy (SLNB) has emerged as the preferred standard procedure in patients with breast cancer, melanoma and other types of cancer. Herein, we developed a method to intra-operatively map SLNs and differentiate tumor metastases within SLNs at the same time, with the aim to provide more accurate and real-time intraoperative guidance. Experimental Design: Hyaluronic acid (HA), a ligand of lymphatic vessel endothelial hyaluronan receptor (LYVE)-1, is employed as a SLN mapping agent after being conjugated with a near-infrared fluorophore (Cy5.5). Different sized HAs (5, 10 and 20K) were tested in normal mice and mice with localized inflammation to optimize LN retention time and signal to background ratio. Cetuximab, an antibody against epidermal growth factor receptor (EGFR), and trastuzumab, an antibody against human epidermal growth factor receptor 2 (HER2), were labeled with near-infrared fluorophore (IRDye800) for detecting metastatic tumors. LN metastasis model was developed by hock injection of firefly luciferase engineered human head neck squamous carcinoma cancer UM-SCC-22B cells or human ovarian cancer SKOV-3 cells. The metastases within LNs were confirmed by bioluminescence imaging (BLI). IRDye800-Antibodies were intravenously administered $24 \mathrm{~h}$ before local administration of Cy5.5-HA. Optical imaging was then performed to identify nodal metastases. Results: Binding of HA with LYVE-1 was confirmed by ELISA and fluorescence staining. HA with a size of $10 \mathrm{~K}$ was chosen based on the favorable migration and retention profile. After sequential administration of IRDye800-antibodies intravenously and Cy5.5-HA locally to a mouse model with $L N$ metastases and fluorescence optical imaging, partially metastasized LNs were successfully distinguished from un-metastasized LNs and fully tumor occupied LNs, based on the different signal patterns. Conclusions: Fluorophore conjugated HA is a potential lymphatic mapping agent for SLNB. Dual-tracer imaging with the combination of lymphatic mapping agents and tumor targeting agents can identify tumor metastases within SLNs, thus may provide accurate and real-time intra-operative guidance to spare the time spent waiting for a biopsy result.
\end{abstract}

Key words: metastasis, sentinel lymph node, optical imaging, hyaluronic acid, EGFR, HER2.

\section{Introduction}

Due to the large cavity of lymph vessels and slow velocity of lymph, the lymphatic system serves as a transport route for disseminating tumor cells, resulting in metastases [1]. Consequently, many types of malignant tumors such as breast cancer, melanoma, and prostate cancer are prone to metastasize first to 
regional lymph nodes (LNs), through tumor associated lymphatic channels [2, 3]. Since sentinel lymph nodes (SLNs) are the nodes that receive direct lymphatic drainage from a primary tumor site [4], the status of SLNs serves as an indicator of prognosis and therapeutic decision-making $[5,6]$. So far, the gold standard to stage the SLNs is lymphadenectomy and histological evaluation, which is invasive and limited by surgical field for nodal sampling and lack of accuracy [7]. With the development of imaging techniques, currently, pre-surgical diagnosis of SLNs is often based on their morphologic change observed by magnetic resonance imaging (MRI) or x-ray computed tomography (CT) $[8,9]$.

For intra-operative guidance, the most common strategy is to locally administer technetium-99m radiocolloid then a blue dye (either patent blue or methylene blue) several hours later. SLNs can be detected by a gamma probe as hot spots and visualized by bright field as blue nodes [10-13]. Although the value of this procedure has been substantiated in numerous clinical studies [11, 12], it has several drawbacks. Radiocolloid requires complicated and restrictive radioactive management norm and scintigraphy or gamma probes suffer from low sensitivity and poor spatial resolution. The blue dye may stain the surgery area into blue, which may cause inconvenience for the surgery [14]. Moreover, these blue dyes are of small molecular size and migrate through lymphatic system rapidly, giving rise to poor accumulation in the SLN and mess up SLNs with LNs of secondary echelon [15].

Recently, intraoperative fluorescent imaging provided a promising alternative for mapping SLNs. Other than the combination of radiocolloid and blue dyes, fluorescence dyes are radio-free, relatively tissue penetrable and visual view clean. For example, indocyanine green (ICG), a FDA approved near-infrared (NIR) dye with good safety profile, has been intensively investigated both preclinically and clinically for SLN mapping $[16,17]$. Similar to the patent blue or methylene blue dye, ICG is also reported as fast migration and with low retention time in SLNs.

One major limitation of the currently available method is incapable of differentiating SLNs with or without tumor metastases. In most cases, both the surgeon and patient have to wait at least 40 minutes for the result of pathological examination. Thus, an ideal lymphatic imaging strategy should have high signal background ratio for clear SLN detection, be able to differentiate tumor metastasis and provide real-time intraoperative guidance. However, it is very challenging to fulfill all the requirements with a single imaging agent [17]. In this study, we overcome this dilemma by synergistically combining a tumor imaging agent with a novel biocompatible lymphatic mapping agent.

For tumor targeting, we conjugated IRDye800 to cetuxiamb and trastuzumab. Cetuximab is a chimeric antibody against EGFR. EGFR is a cell-surface tyrosine kinase receptor that regulates cellular migration, adhesion, differentiation, and survival and proliferation by controlling downstream MAPK, Akt and JNK pathways [18-20]. Its overexpression widely occurs in solid tumors like head and neck squamous cell carcinoma and breast cancer, in association with tumor progression and metastasis [21, 22]. Trastuzumab targets to HER2, which is overexpressed in around $30 \%$ of breast cancer patients and confers a significantly worse prognosis. Over-expression of HER2 is also known to occur in ovarian, stomach, and aggressive forms of uterine cancer [23]. For lymphatic mapping, we chose hyaluronic acid (HA) due to the tunable size and excellent biocompatibility. Moreover, HA is the substrate of LYVE-1, which is expressed predominantly on lymphatic endothelium [24]. The binding of Cy5.5 labeled HA to LYVE-1 will increase the retention of the imaging probe to provide optimal time window for surgery guidance. In this study, we hypothesize that with dual-tracer optical imaging, not only the SLNs can be mapped but also the tumor metastases within SLNs can be identified at the same time (Fig. 1).

\section{Materials and Methods}

\section{HA labeling and anti-EGFR antibody labeling}

HAs with different molecular weights of 5, 10 and $20 \mathrm{kDa}$, were obtained from Lifecore Biomedical LLC (MN, USA) (Cat\#: HA5K-1, HA10K-1 and HA20K-1). All HAs were used as received without further purification. Cyanine 5.5 amine (Cy5.5) was purchased from Lumiprobe Corporation (FL, USA) (Cat\#: 470C0). N-Hydroxysuccinimide (NHS), $\mathrm{N}$-(3-Dimethylaminopropyl)-N'-ethylcarbodiimide hydrochloride (EDC) and Triethylamine (TEA) were obtained from Sigma-Aldrich (MO, USA) and used without further purification. The following method was employed to conjugate Cy5.5 to HA: $10 \mathrm{mg}$ HA (in different molecular weights) was dissolved in 150 $\mu \mathrm{l} \mathrm{MilliQ}$ water followed by adding $150 \mu \mathrm{L}$ DSO with vigorous vortex to obtain a clear solution. In the case of $20 \mathrm{kDa} \mathrm{HA}$, the solution became viscous. A heating chamber was used to gently warm up the solution to $50{ }^{\circ} \mathrm{C}$ until the solution became clear liquid. $15 \mu \mathrm{l}$ Cy5.5 amine ( $33 \mathrm{mg} / \mathrm{ml}$ in DMSO) was added into the HA solution. $11 \mathrm{mg}$ NHS and $5 \mathrm{mg}$ EDC were separately weighted. $100 \mu \mathrm{l}$ DMSO was used to dissolve the NHS/EDC powder which was 
then added into the HA and Cy5.5 mixture solution with brief vigorous vortex. $4 \mu$ TEA $(1 \% \mathrm{v} / \mathrm{v})$ was added into the mixture solution to catalyze the reaction. The solution was then incubated on a shaker at $1000 \mathrm{rpm}$ at room temperature overnight. The reaction product was purified three times using PD-10 columns (GE Healthcare Life Sciences, PA, USA). The Cy5.5-HA conjugate fraction was collected and subjected to lyophilization to yield dry products in bright blue color. The yield is about $70 \%$ by weight.

As a negative control to $\mathrm{HA}$, we used the branched 6-armed poly (ethylene glycol) (6-armed
PEG) (M.W.= $10 \mathrm{kDa})($ SunBio, Cat\# P6AM-10) in the study. The PEG is amine terminated. We hence used Cy5.5-NHS (Cy5.5 NHS) for the conjugation reaction. $10 \mathrm{mg}$ 6-armed PEG was dissolved in $150 \mu \mathrm{l}$ MilliQ water followed by adding $150 \mu \mathrm{l}$ DMSO. $10 \mu \mathrm{l}$ Cy5.5 NHS $(20 \mathrm{mg} / \mathrm{ml}$ in DMSO) was added into the PEG solution. $3 \mu \mathrm{l}$ TEA $(1 \% \mathrm{v} / \mathrm{v})$ was added into the mixture solution. The reaction was carried out on a shaker at $1000 \mathrm{rpm}$ at room temperature overnight. The purification procedure was identical to that for HA-Cy5.5 conjugate. The yield is about $80 \%$ by weight.
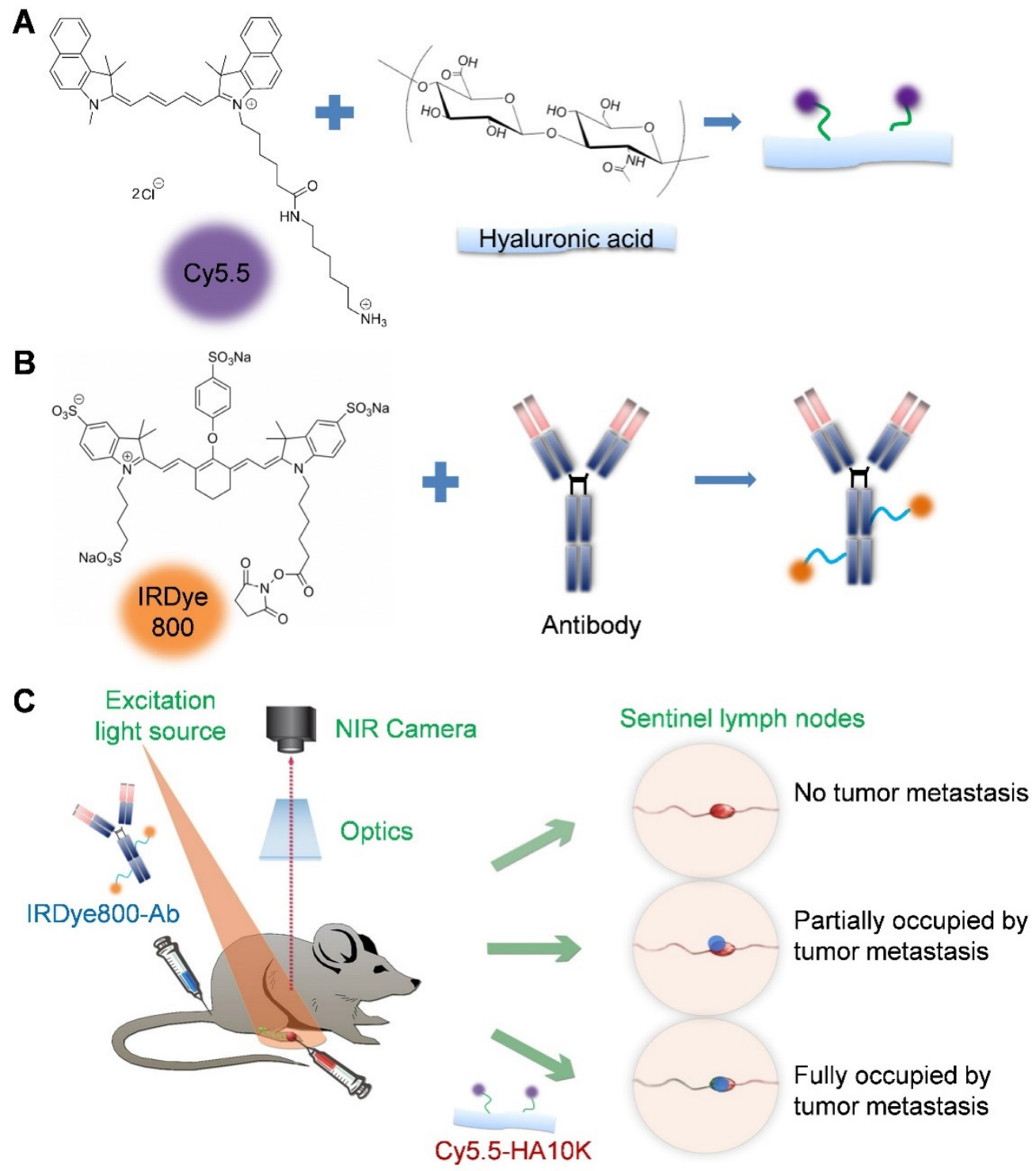

Figure 1. (A) Conjugation of hyaluronic acid (HA) with Cy5.5 (excitation $675 \mathrm{~nm}$, emission $694 \mathrm{~nm}$ ). (B) Conjugation of antibody with IRDye800 (excitation $770 \mathrm{~nm}$, emission $786 \mathrm{~nm}$ ). (C) Schematic of dual-tracer fluorescence optical imaging. The IRDye800 conjugated antibodies were injected via tail vein $24 \mathrm{~h}$ before local administration of Cy5.5-HA. Fluorescence imaging was performed $60 \mathrm{~min}$ later with a near-infrared filter set (excitation $704 \mathrm{~nm}$, emission $745 \mathrm{~nm}$ longpass). The signals from Cy5.5-HA and IRDye800-mAb were then unmixed based on their unique spectra to reflect tumor metastasis within the sentinel lymph node. 
Pharmaceutical grade cetuximab and trastuzumab antibodies were used to target EGFR and HER2 on the lymph node metastatic cancer cells, respectively. To separate the antibody signal from Cy5.5-HA, we used IRDye800CW NHS ester (IRDye800) (LI-COR Biotechnology, NE, USA Cat\# 929-70021) to label the amine group of lysine residues from the antibodies. Briefly, $500 \mu \mathrm{l}$ antibodies (2 $\mathrm{mg} / \mathrm{ml}$ in saline as received) was mixed with $4.5 \mu \mathrm{l}$ IRDye800 (5 mg/ml in DMSO) followed by brief vortex to obtain homogenous solution. The reaction was carried out on a benchtop shaker at $1000 \mathrm{rpm}$ at room temperature for $1.5 \mathrm{~h}$. PD-10 column was used to purify the reaction product. To maximally retain the antibody binding affinity after dye labeling, we optimized the antibody to IRDye800 molar ratio at 1:3.

\section{HA binding assay}

To test HA binding with LYVE-1, 96-well plates were first coated overnight with mouse LYVE-1 protein (R\&D Systems) or control BSA $(0.5-8 \mu \mathrm{g} / \mathrm{mL}$ in PBS). The wells were then incubated with Cy5.5-HA10K (1 $\mathrm{nmol} / \mathrm{mL}$ of Cy5.5) in PBS containing $0.05 \%$ Tween 20 for $2 \mathrm{~h}$. After each step, the wells were washed gently for $5 \mathrm{~min}$ for 5 times with PBS containing $0.05 \%$ Tween 20 . The results were observed by A Maestro II small animal optical imaging system (Cambridge Research \& Instrumentation) with an orange filter (excitation: 605 $\mathrm{nm}$; emission: $645 \mathrm{~nm}$ long pass).

\section{Animal models}

All animal studies were conducted in accordance with the principles and procedures outlined in the Guide for the Care and Use of Laboratory Animals [25] and were approved by the Institutional Animal Care and Use Committee of the Clinical Center, NIH. UM-SCC-22B human head and neck carcinoma cancer cells and SKOV-3 human ovarian cancer cells were genetically labeled with a firefly luciferase reporter gene and were cultured as previously reported [26]. 1 $\times 10^{5} \mathrm{fLuc}^{+} \mathrm{UM}-\mathrm{SCC}-22 \mathrm{~B}$ or SKOV-3 cells in $10 \mu \mathrm{L}$ PBS were injected into the right hock of 6-week-old athymic nude mice. The mice were monitored by bioluminescence imaging (BLI) once a week to evaluate tumor proliferation and metastases using a Lumina II imaging system (Caliper Life Sciences). 150 $\mathrm{mg} / \mathrm{kg}$ D-luciferin in $100 \mu \mathrm{L}$ of normal saline was administrated via intraperitoneal injection and mice were imaged $10 \mathrm{~min}$ after. Mice were kept anesthetized by $2 \%$ isoflurane during the imaging process.

\section{Fluorescence optical imaging}

A Maestro II small animal optical imaging system (Cambridge Research \& Instrumentation) was employed for optical imaging. The mice were maintained under anesthesia with 1.5 to $2 \%$ isoflurane in oxygen during injection and imaging. For Cy5.5 labeled HAs and PEG, an orange filter set with excitation of $605 \mathrm{~nm}$ and emission of $645 \mathrm{~nm}$ longpass (540-610 nm) was utilized. The probes $(0.2 \mathrm{nmol}$ of Cy5.5 in $20 \mu \mathrm{L}$ ) were injected into right hock of mice and the mice were imaged every $10 \mathrm{~min}$. For dual-probe imaging, IRDye800 labeled antibodies (1 nmol of dye in $150 \mu \mathrm{L}$ ) was injected via tail vein $24 \mathrm{~h}$ before local injection of Cy5.5-HA10K (0.2 nmol of Cy5.5 in $20 \mu \mathrm{L}$ ). Optical imaging was performed at 60 min after Cy5.5-HA10K injection using a NIR filter set (excitation $704 \mathrm{~nm}$, emission $745 \mathrm{~nm}$ longpass). The signals from Cy5.5 and IRDye800 were unmixed based on their specific spectra using the software provided by the manufacturer (CRI, Maestro).

\section{Immunohistology}

At $2 \mathrm{~h}$ after Cy5.5-HA10K injection, popliteal LNs were harvested and were sectioned into $10 \mu \mathrm{m}$ slices by using an Ultrapro 5000 cryostat (Vibratome). Slices were fixed with Z-fix solution for $15 \mathrm{~min}$ and then were blocked with PBS containing 1\% BSA for 1 $h$. Slices were incubated with rat anti-mouse LYVE-1 primary antibody (1:400, MBL International Corporation) at room temperature for $2 \mathrm{~h}$, and then with Cy3-conjugated donkey anti-rat secondary antibody (1:200; Jackson ImmunoResearch Laboratories). Between each step, the slices were gently washed 5 times with $0.05 \%$ tween 20 PBS solution (PBST) for 5 min each time. The slices were then incubated with medium containing 4',6-diamidino-2-phenylindole (DAPI). The staining observation was done with an epifluorescence microscope (X81; Olympus). H\&E staining was performed as previously reported [27].

\section{Data quantification and statistics}

BLI and fluorescent images were analyzed by Living image ${ }^{\circledR}$ (PerkinElmer) and Maestro (version 2.10.0, PerkinElmer), respectively. Fluorescence intensity was measured by drawing regions of interest (ROIs) over LNs. All quantitative data were represented as mean \pm SD. Statistical analysis was performed with Graphpad (version 4.0, GraphPad Software, Inc.). Student $t$ test was used for two-group comparisons at different time points. $\mathrm{P}<0.05$ was considered statistically significant.

\section{Results}

\section{HA binds specifically with LYVE-I}

LYVE-1 is a hyaluronic acid receptor with sequence similar to CD44 expressed on lymphatic endothelial cells. Anti-LYVE-1 immunostaining 
confirmed that extensive lymphangiogenesis happened in the tumor-draining $\operatorname{LN}[28,29]$. The binding to LYVE-1 will facilitate the migration and retention of HA based probes for effective lymphatic mapping. We first confirmed the binding between HA and LYVE-1 in vitro via ELISA assay. With increased concentration of LYVE-1 protein coated in the well, higher fluorescence signal from Cy5.5-HA was observed. While in control wells coated with bovine serum albumin (BSA), the fluorescence intensity of Cy5.5-HA remained unchanged (Fig. 2A and 2B).

To further demonstrate the specific binding of Cy5.5-HA to LYVE-1, popliteal LNs were sectioned and stained with an antibody against LYVE-1 and Cy5.5-HA. As shown in Fig. 2C, although C5.5-HA showed more diffusive distribution than that of LYVE-1 antibody, colocalization of LYVE-1 and Cy5.5-HA can be recognized on the overlaid images, indicating the binding of HA to LYVE-1 within the lymph node. The potential toxicity of Cy5.5-HA10K was evaluated by MTT assay. No apparent cytotoxicity was observed when the Cy5.5-HA10K concentration was as high as $4.0 \mathrm{mg} / \mathrm{ml}$ (Fig. S1).

\section{Optimization of Cy5.5-HA with different sizes for lymphatic mapping}

Besides specifically binding with lymphatic maker, the size of the imaging probe is also critical for migration and retention. Based on experience from
99mTc-labeled colloid, the optimal particle size is from 50 to $200 \mathrm{~nm}$ since the radioactive colloids are cleared by lymphatic drainage with a speed that is inversely proportional to the particle size after interstitial injection [11]. In the next experiment, we assessed Cy5.5-HA of different sizes (5K, $10 \mathrm{~K}$ and $20 \mathrm{~K})$ with the aim to optimize the imaging probe for SLN mapping. Cy5.5-HAs were intradermally injected into the hind footpads of normal FVB mice and longitudinal fluorescence optical imaging was carried out to record the migration and retention of the imaging tracers. As revealed by the images (Fig. 3A), the lymphatic vessels and LNs along the path (popliteal LNs and sciatic LNs) can be clearly visualized with low background signal. Cy5.5-HAs migrated to the popliteal LN in a size-dependent manner. The popliteal LNs were clearly identified with Cy5.5-HA5K within $30 \mathrm{~min}$ after injection but not with Cy5.5-HA20K until after $1 \mathrm{~h}$. At late time points, the fluorescence signal migrated out of popliteal LNs to the sciatic LNs, especially with $5 \mathrm{~K}$ and $10 \mathrm{~K}$ Cy5.5-HAs. It corresponded to our hypothesis that HA can be bound in the first drain LNs and therefore can be a good SLN mapping agent. Due to fast migration with Cy5.5-HA5K and tardy emerging of popliteal LN with Cy5.5-HA20K, Cy5.5-HA10K was chosen in the subsequent experiments (Fig. 3B and 3C).
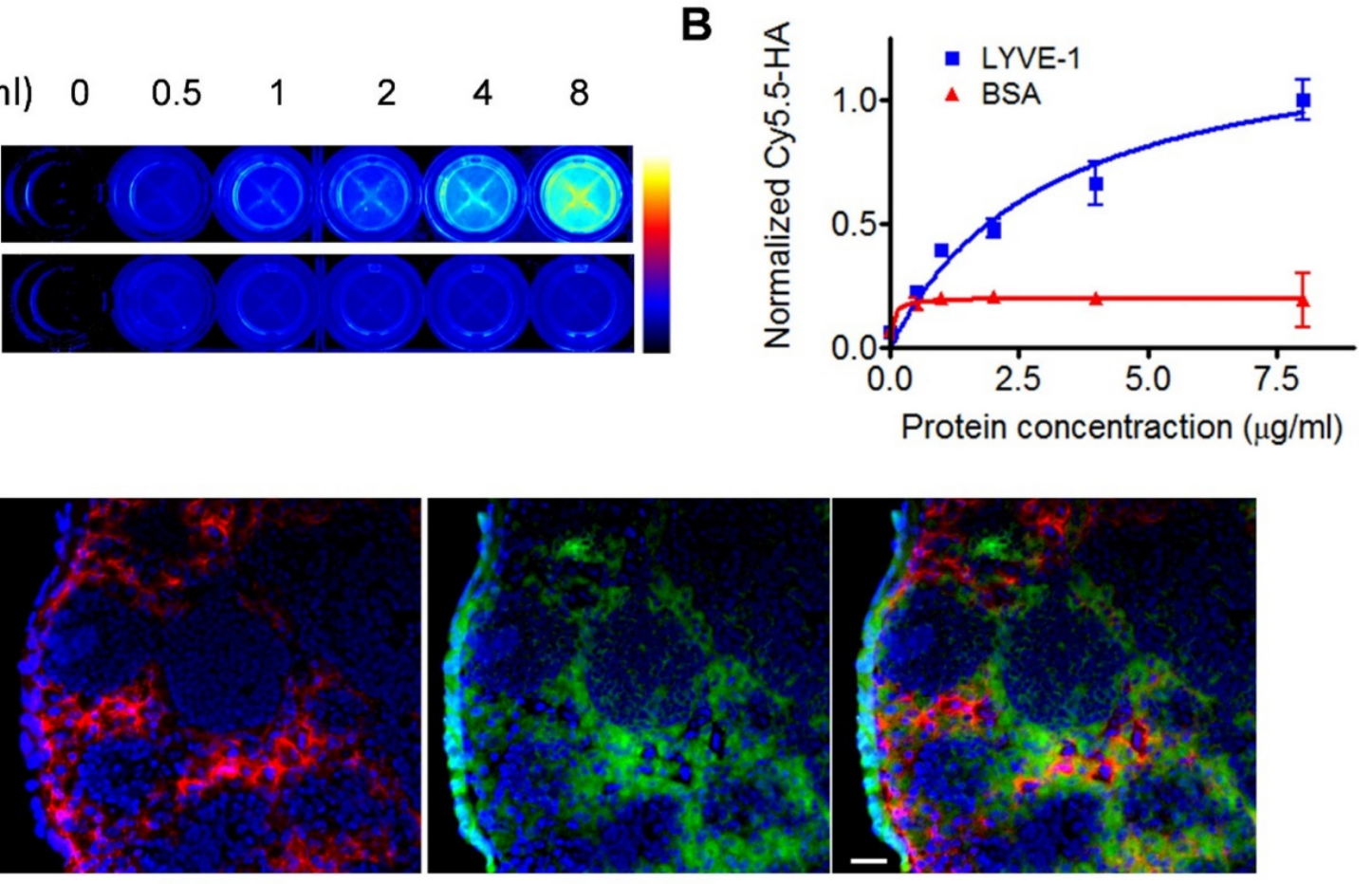

LYVE-1
Overlay

Figure 2. In vitro characterization of HA binding to LYVE-1. (A) LYVE-1 and bovine serum albumin of different concentrations were coated on 96-well plates and Cy5.5-HA10K was added for incubation. After washing, the plates were visualized by fluorescence images. (B) Quantification of Cy5.5-HA binding to LYVE-1 proteins and BSA. (C) At $2 \mathrm{~h}$ after Cy5.5-HA10K injection, popliteal LNs were harvested and sectioned into $10 \mu \mathrm{m}$ slices. Fixed slices were then stained against LYVE-1. Co-localization of LYVE-1 (red) and HA (green) was observed. Nuclei were stained as blue with DAPI. The scale bar is $20 \mu \mathrm{m}$. 
A

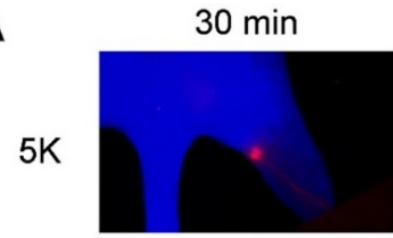

$10 \mathrm{~K}$
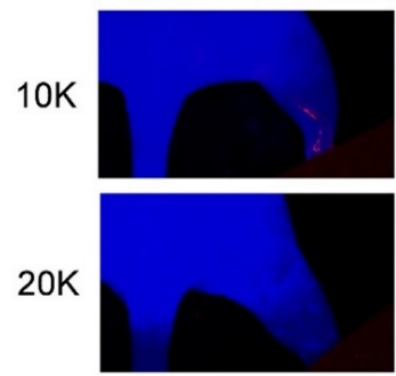

B

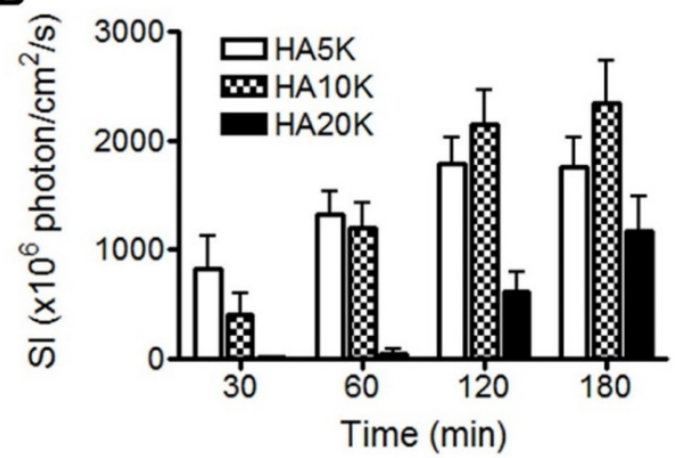

$60 \mathrm{~min}$
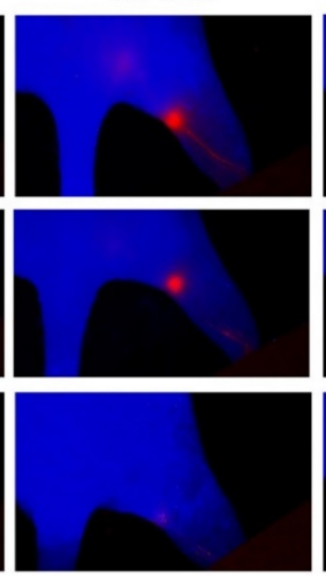

C
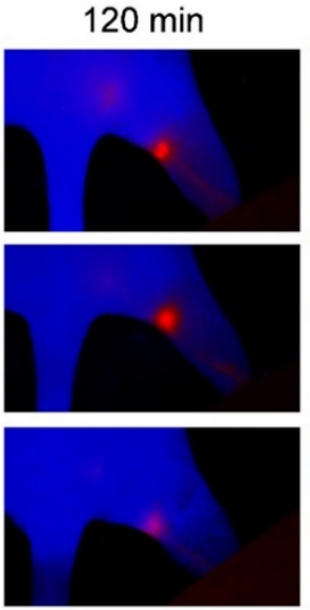
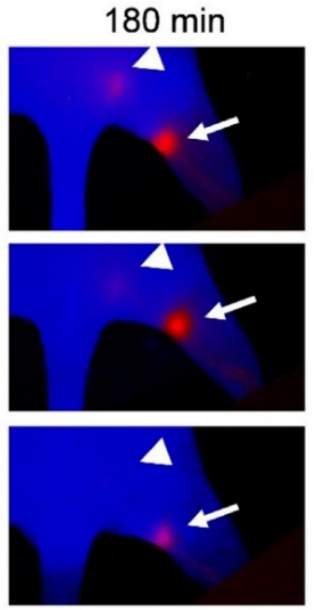

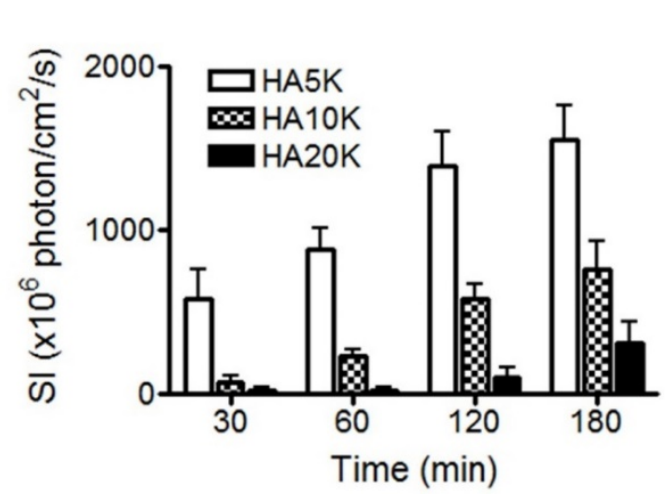

Figure 3. Comparison of Cy5.5-HA of different sizes as to migration and retention. (A) Representative fluorescent images after intradermal injection of $5 \mathrm{~K}$, $10 \mathrm{~K}$, or $20 \mathrm{~K}$ Cy5.5-HA into the right paw. The images were acquired at different times after Cy5.5-HA injection using an orange filter set (excitation $605 \mathrm{~nm}$, emission $645 \mathrm{~nm}$ longpass). Popliteal (arrows) and sciatic (triangles) LNs were visualized at different times in a size-dependent manner. Quantification of signal intensity from popliteal (B) and sciatic (C) LNs.

As aforementioned, we hypothesized that binding with LYVE-1 would increase the retention of Cy5.5-HA within LNs. To further confirm this, Cy5.5-HA10K was compared with Cy5.5-PEG10K. As shown in Fig. 4, Cy5.5-PEG10K showed much faster migration rate than Cy5.5-HA10K, with both popliteal and sciatic LN visualized within $30 \mathrm{~min}$ after local injection. The ratio of signal intensity from sciatic LN to popliteal LN (RSP) was introduced to provide a quantitative comparison (Fig. 4B). Significantly lower RSPs from mice received Cy5.5-HA10K were observed at all the time points examined $(30,60$ and $120 \mathrm{~min})$, compared with those from mice received Cy5.5-PEG10K. These results implied that the prolonged retention of Cy5.5-HA10K is not only size dependent but also affected by LVYE-1 binding, resulting in a high contrast identification of SLNs.

We also applied Cy5.5-HA10K to an inflammation model, which was induced by local intramuscular injection of turpentine oil [30]. The inflammatory reaction with LNs was indicated by swollenness on MRI. The inflamed LNs showed much higher signal intensity than the co-lateral LNs (Fig. S2). Moreover, the signal intensity of LNs was much stronger after the skin was removed.

\section{Tumor detection by IRDye $\mathbf{8 0 0}$-cetuximab}

Cetuximab has been labeled with various radioisotopes and fluorophores for EGFR positive tumor imaging [18]. After intravenous injection of IRDye800-cetuximab, the subcutaneous tumors can be clearly visualized at $4 \mathrm{~h}$ and the strong signal lasts up to $96 \mathrm{~h}$ (Fig. 5A). The tumor/background ratio reached a plateau between 24 and $96 \mathrm{~h}$ (Fig. 5B and 5C). The long-lasting signal may not facilitate repetitive imaging but gives a convenient time window for intraoperative imaging of tumor metastases. We further evaluated the detection limit of the antibody based optical imaging. First, we incubated IRDye800-cetuximab with UM-SCC-22B cells then inoculated nude mice with the cells subcutaneously. As shown in Fig. S3, 1000 cells can be easily visualized from apparent auto-fluorescence signal come out of the skin. We also inoculated subcutaneously unlabeled cells first to nude mice. After $24 \mathrm{~h}$, IRDye800-cetuximab was administered through tail vein (Fig. 5D and 5E). Even with the auto-fluorescence from the skin, the nodules of 1000 cells can be clearly identified. With 300 cells, the signal from the cells is not strong enough to be separated from background. 
A

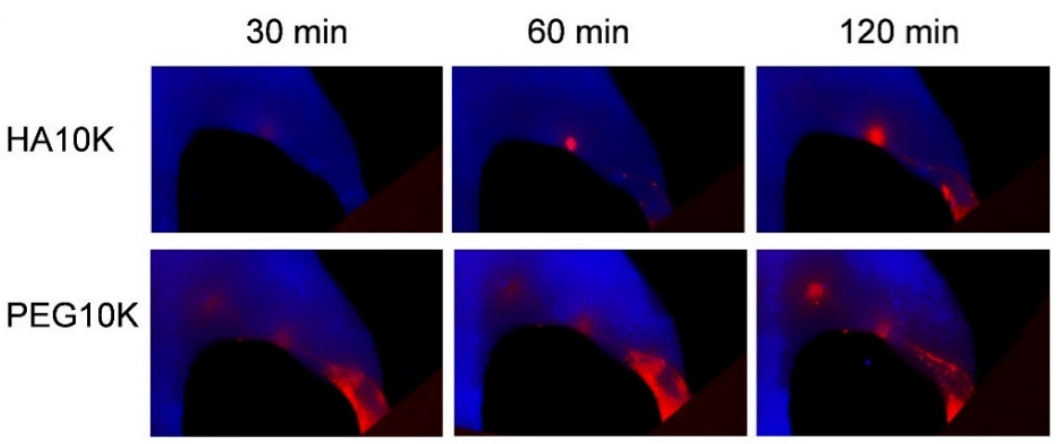

B

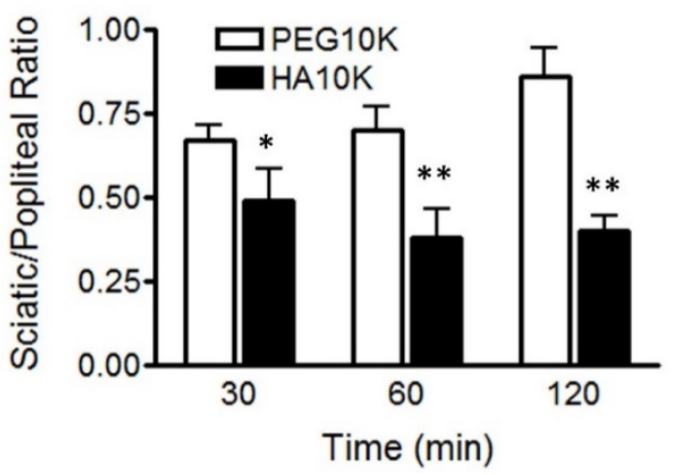

Figure 4. Comparison of Cy5.5-HA10K and Cy5.5-PEG10K. (A) Representative images of Cy5.5-HA10K and Cy5.5-PEG10K at 30, 60 and 120 min after hock injection. Compared with Cy5.5-HA10K, Cy5.5-PEG10K showed less retention in popliteal LNs and faster migration into sciatic LNs. (B) Sciatic to popliteal ratio (SPR) at 30, 60, and 120 min after imaging agent administration. SPR of Cy5.5-HA10K is significantly lower than that of Cy5.5-PEG10K at all the time points $(*, P<0.05$; **, $P<0.01)$.

A

$\mathrm{Oh}$

$1 \mathrm{~h}$

$4 \mathrm{~h}$ $8 \mathrm{~h}$ $16 \mathrm{~h}$ $24 \mathrm{~h}$ $48 \mathrm{~h}$ $96 \mathrm{~h}$
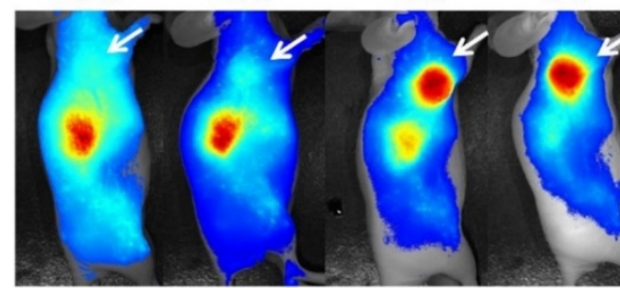

B

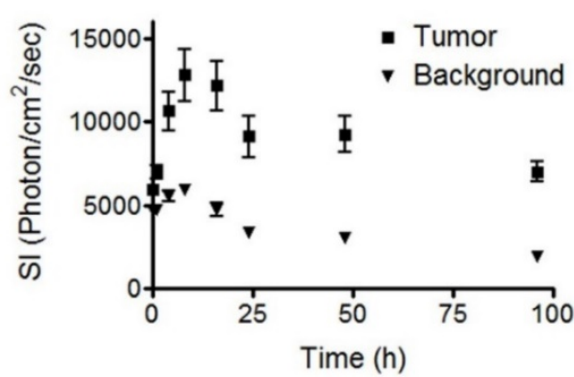

D

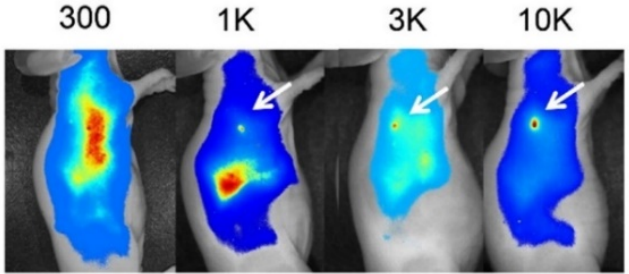

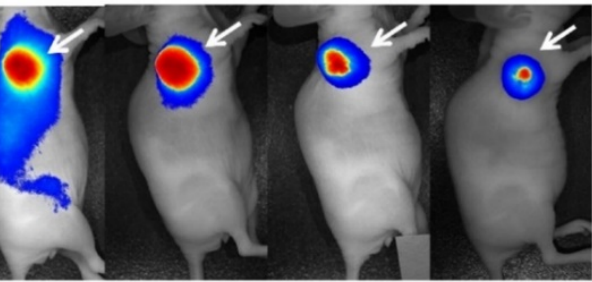

C

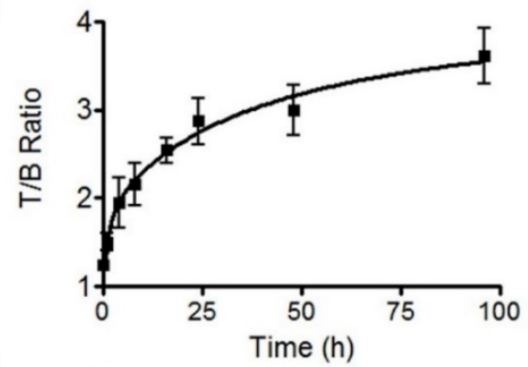

E

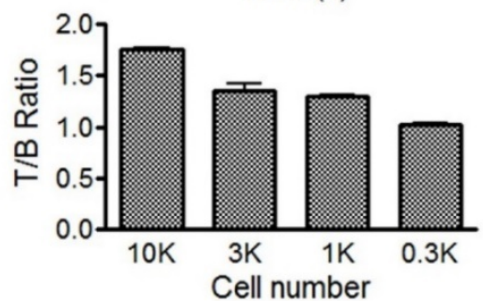

Figure 5. Fluorescence optical imaging of UM-SCC-22B tumors and cell clusters using IRDye 800 -cetuximab. (A) Longitudinal fluorescence imaging of subcutaneous UM-SCC-22B xenografted tumors. Each animal received around $1 \mathrm{nmol}$ of IRDye800-centuximab via tail vein. The tumors (white arrows) were clearly visualized from $4 \mathrm{~h}$ to 96 $\mathrm{h}$ after injection. (B) Quantitative analysis of fluorescence intensity within tumor and background area. The leg muscle was chosen as the background. (C) Quantitative analysis of tumor to background ratio (TBR). (D) Evaluation of sensitivity of IRDye800-cetuximab in tumor cell detection. Different amount UM-SCC-22B cells were inoculated subcutaneously in nude mice $(n=4)$. Twenty-four hours after tumor cell inoculation, IRDye800-cetuximab $(1 \mathrm{nmol}$ of dye in $150 \mu \mathrm{L})$ was injected via tail vein. Another $24 \mathrm{~h}$ later, optical imaging was performed using a NIR filter set (excitation $704 \mathrm{~nm}$, emission $745 \mathrm{~nm}$ longpass). The cell cluster containing $1 \mathrm{~K}$ cells were clearly visualized. (E) Quantitative analysis of ratio of signal intensity from tumor cell clusters to background area. 


\section{Mapping sentinel lymph node metastasis}

To develop LN tumor metastasis model, $0.1 \times 10^{6}$ FLuc $^{+}$UM-SCC-22B or FLuc ${ }^{+}$SKOV-3 cells in $10 \mu \mathrm{L}$ of PBS were injected into the hock region. Bioluminescence imaging was performed weekly to monitor the tumor proliferation and metastasis. Tumor metastases within popliteal LNs were visualized at week 6 for UM-SCC-22B cells and week 7 for SKOV-3 cells (Fig. S4 and Fig. S5).

Due to the different emission wavelengths between Cy5.5 and IRDye800, the fluorescence signals can be readily separated (Fig. S6). In order to mimic clinical situation, dual-imaging fluorescence optical imaging was performed at different stages of tumor metastases. IRDye800-antibodies were administered intravenously $24 \mathrm{~h}$ before local administration of Cy5.5-HA10K. As demonstrated in Fig. 6 and Fig. S7, three typical patterns of fluorescence signal distribution within LNs could be observed. Without tumor cell invasion and metastasis, the whole LN was presented with signal from Cy5.5-HA10K only and no signal from IRDye800-cetuximab or trastuzumab was found in LNs. With tumor cell invasion and SLN partially occupied by metastatic tumor cells, Cy5.5-HA10K can still migrate to LNs through remaining lymphatic channels, and metastatic tumor

A

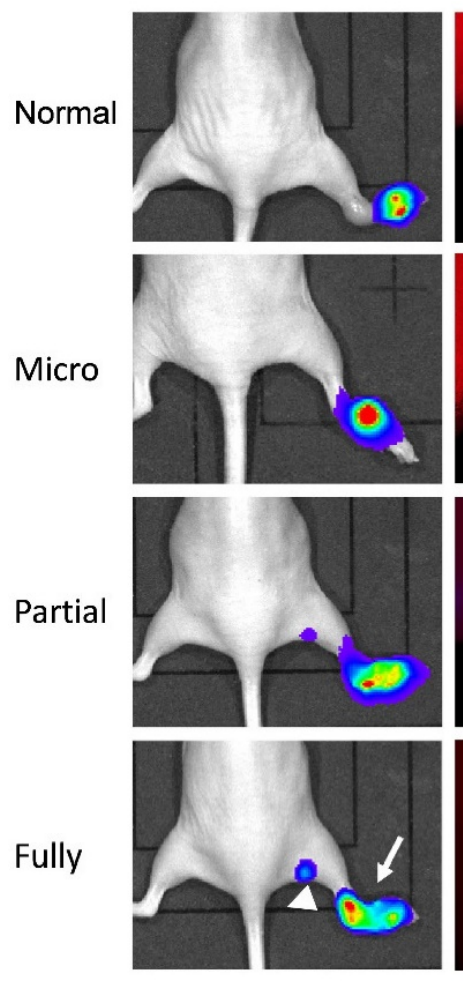

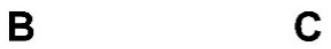
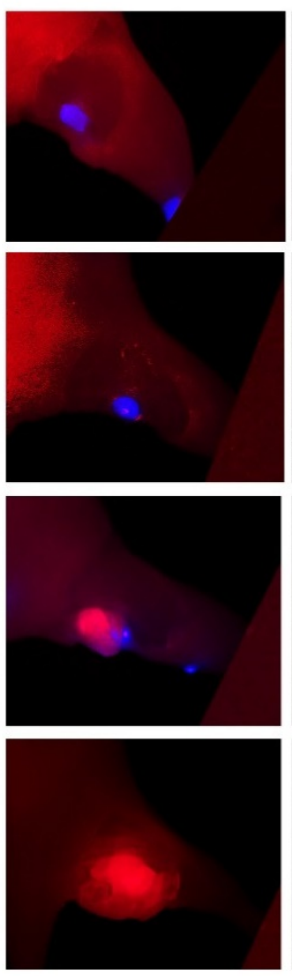

C
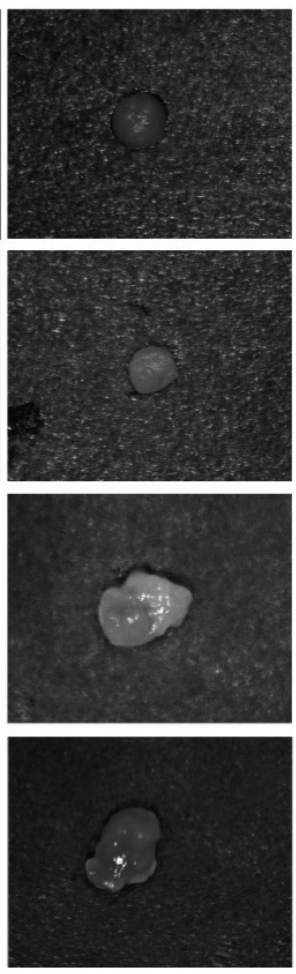

cells can be visualized by IRDye800-cetuximab or trastuzumab. Once the LNs were fully occupied by metastatic tumor and lymphatic circulation was completely obstructed, only signal from IRDye 800-cetuximab or trastuzumab can be observed with no signal from Cy5.5-HA10K in the SLNs.

\section{Discussion}

As an alternative to axillary lymph node dissection (ALAD), lymphatic mapping with sentinel lymph node biopsy (SLNB) has emerged as an effective method to detect axillary metastases without adverse long-term side effects. Although still debatable, the clinical advantages of SLNB over ALND are apparent, and the procedure is becoming the preferred standard in patients with breast cancer or melanoma [31]. Moreover, SLNB has become established clinical practice in patients with other types of cancer including penile, anal, colorectal and prostate cancer [32]. SLNs can be identified with the help of various lymphatic mapping agents including radiolabeled colloid, blue dyes, and nanoparticles. Especially recently, various nanoparticles have been investigated for lymphatic imaging due to easily tunable size and strong signal intensity [17] [33-35].

Figure 6. Mapping UM-SCC-22B tumor metastasis in sentinel lymph nodes by dual-tracer fluorescence optical imaging. (A) LN metastasis model was developed by hock injection of Fluc ${ }^{+}$UM-SCC-22B cells and the metastases were confirmed by BLI. The original tumor sites were pointed by arrows and tumor metastasized LNs by arrow heads. (B) IRDye800-cetuximab (anti-EGFR antibody) was injected intravenously at $24 \mathrm{~h}$ before imaging and Cy5.5-HA10K was administered locally at $1 \mathrm{~h}$ before imaging with a Maestro II optical imaging system. The fluorescence signal was unmixed based on the corresponding specific spectra from Cy5.5 (designated as blue) and IRDye800 (designated as red). The skin was peeled to mimic the operative setting. (C) Bright field and (D) optical imaging of excised LNs. LN without tumor invasion showed red color only from Cy5.5-HA. Partially tumor invaded LN showed mixed signals from both Cy5.5-HA10K and IRDye800-cetuximab. Fully tumor occupied LN showed signal only from IRDye800-cetuximab since the lymphatic vessels were blocked by tumor tissue. (E) H\&E staining of corresponding LNs. T, tumor tissue; LN, lymphatic tissue. 
Although the preclinical results are very promising, majority of these probes are composed of heavy metals and clinical translation may be hindered by the perceived acute and chronic toxicity [36]. Thus, in this study, we chose HA as the lymphatic mapping agent after labeling with the near-infrared dye Cy5.5 (excitation 675nm, emission $694 \mathrm{~nm}$ ). There are several advantages to use HA as the lymphatic mapping agent. First, it is completely biocompatible due to the existence of endogenous HAs. Second, the size of HA is tunable. More importantly, the specific binding of HA to LYVE-1 receptors on the lymphatic endothelial cells can further increase the retention of HA probe within the lymph nodes. The long retention within LNs would facilitate a manageable surgery time window without diffusing to the second-echelon LNs. Among HAs with different sizes, the solution of 20K HA becomes viscous thus the signal in the first line LNs is still pretty weak at $60 \mathrm{~min}$ after injection. HA5K migrated rapidly to LNs of the second echelon (Fig. 3). HA10K showed proper migration and relatively long SLN retention time so it was chosen for further evaluation.

For most of lymphatic mapping agents, it is very challenging to identify tumor invasion to the SLNs detected on the images. With superparamagnetic nanoparticles, Harishinghani et al. [37] revealed that high-resolution magnetic resonance imaging (MRI) could identify small nodal metastases in patients with prostate cancer. However, this strategy may not be practical to provide intraoperative guide for SLN identification and characterization. Instead, fluorescence optical imaging provides more convenient setup for mapping SLNs. Low penetration and scattering of photons will be overcome to a great extent due to direct exposure of the surgical field [16]. However, when the lymph node is fully occupied by metastasized tumors or the lymphatic vessels are blocked by tumor tissue, locally administered imaging agent cannot migrate through for LN detection. Therefore, in this study, we developed dual-tracer optical imaging to combine lymphatic mapping agents and tumor targeting agents, with the aim to provide a real-time and accurate evaluation of SLNs. Using Cy5.5-HA10K as the lymphatic mapping agent and IRDye800 conjugated EGFR and HER2 antibodies as the tumor targeting agents, the tumor draining LNs can be clearly visualized.

More importantly, the different patterns of signal distribution within LNs could reveal the progress of tumor metastases. Without tumor invasion, LNs can be identified by Cy5.5-HA. When tumor cells invade to LNs, they can be visualized by NIR dye labeled antibodies to reorganize the specific tumor makers on the cell surface. In addition, the signal intensity is proportional to tumor burden within LNs. At the same time, the residual LN tissue can be detected by Cy5.5-HA. When the metastasized tumor fully occupies the LNs or block the lymphatic vessels for Cy5.5-HA migration, only signal from the antibodies can be recorded. In this study, we focused on detection and staging of LNs so no imaging of the primary tumors was evaluated. The IRDye 800 conjugated antibodies may be helpful to identify the border line of the primary lesions during the surgical operation [38].

Lymphatic mapping agent HA can be used in all types of tumors for SLN detection. However, no universal tumor targeting agent would be available due to the diversity and heterogeneity of malignant diseases [39]. EGFR and HER2 targeted antibodies were used in this study since both EGFR and HER2 are established tumor makers for cancer of various types [23, 40]. For prostate cancer, various prostate-specific membrane antigen (PSMA) targeted probe may be used [41]. In the clinic, the imaging target can be determined based on the pre-surgery biopsy of the primary tumor, although the diversity of cell surface marker may exist between primary lesions and LN metastases [42]. Antibody based imaging probe may have advantage over those with relatively small molecular sizes for dual-tracer optical imaging, mainly due to their long retention time, which can provide a wide time window for the surgery operation. For tumor metastases detection, the specificity can be guaranteed by the specific binding of the antibodies to the tumor cell surface marker. Consequently, positive signal would be a reliable indicator of tumor invasion. The sensitivity is more important since microscopic lesions may be ignored with optical imaging. The results demonstrated that as few as one thousand cells can be recognized with optical imaging. However, intact skin cause relatively high background signal. In addition, the tumor cell cluster does not have enough blood supply for imaging probe delivery. Taking all these into consideration, our results may underestimate the sensitivity of optical imaging using NIR dye labeled antibodies for micro-metastases detection. All these data warrant further clinical translation to evaluate the sensitivity and specificity of this method in evaluating tumor metastases within sentinel LNs.

It is worth noting that the tumor metastasis model was established using immunodeficient nude mice. It has been reported that the structure and cell population of the lymph nodes in the athymic nude mice are different from those of immunocompetent mice $[43,44]$. The variance of morphology may cause misinterpretation of the status of LNs based on MRI or CT [44]. Our results indicated that LNs in both 
immunocompetent and immunodeficient mice could be detected using Cy5.5-HA10K as the optical imaging probe since the signal intensity is independent of morphology of LNs. In the animal model, the optimal imaging window for Cy5.5-HA10K is from $60 \mathrm{~min}$ to $180 \mathrm{~min}$. However, the lymphatic drainage basin in the mouse model is single so the flow between the nodes is sequential. Due to increased complexity in the clinic, the optimization of the size of imaging probe and time window for imaging may be required.

In summary, we validated that $\mathrm{Cy} 5.5-\mathrm{HA} 10 \mathrm{~K}$ is an effective imaging agent for SLN mapping due to its long SLN retention. By combining the tumor targeting agents and lymphatic mapping agents, the dual-tracer optical imaging can not only locate the SLNs, but also evaluate tumor metastases with SLNs. This strategy provides accurate and real-time intra-operative guidance to spare the time spent waiting for a biopsy.

\section{Supplementary Material}

Supplementary figures.

http://www.thno.org/v07p0153s1.pdf

\section{Acknowledgment}

The research was supported by the Intramural Research Program, National Institute of Biomedical Imaging and Bioengineering, National Institutes of Health.

\section{Competing Interests}

The authors have declared that no competing interest exists.

\section{References}

1. Wong SY, Hynes RO. Lymphatic or hematogenous dissemination: how does a metastatic tumor cell decide? Cell Cycle. 2006; 5: 812-7.

2. Picker LJ, Butcher EC. Physiological and molecular mechanisms of lymphocyte homing. Annu Rev Immunol. 1992; 10: 561-91.

3. Karaman S, Detmar M. Mechanisms of lymphatic metastasis. J Clin Invest. 2014; 124: 922-8.

4. Uren RF, Howman-Giles R, Thompson JF, Shaw HM, Quinn MJ, O'Brien CJ, et al. Lymphoscintigraphy to identify sentinel lymph nodes in patients with melanoma. Melanoma Res. 1994; 4: 395-9.

5. Tammela T, Alitalo K. Lymphangiogenesis: Molecular mechanisms and future promise. Cell. 2010; 140: 460-76.

6. Veronesi U, Paganelli G, Galimberti V, Viale G, Zurrida S, Bedoni M, et al. Sentinel-node biopsy to avoid axillary dissection in breast cancer with clinically negative lymph-nodes. Lancet. 1997; 349: 1864-7.

7. Davis GL. Sensitivity of frozen section examination of pelvic lymph nodes for metastatic prostate carcinoma. Cancer. 1995; 76: 661-8.

8. Torabi M, Aquino SL, Harisinghani MG. Current concepts in lymph node imaging. J Nucl Med. 2004; 45: 1509-18.

9. Qian CN, Berghuis B, Tsarfaty G, Bruch M, Kort EJ, Ditlev J, et al. Preparing the "soil": the primary tumor induces vasculature reorganization in the sentinel lymph node before the arrival of metastatic cancer cells. Cancer Res. 2006; 66: 10365-76.

10. Wilhelm AJ, Mijnhout GS, Franssen EJ. Radiopharmaceuticals in sentinel lymph-node detection - an overview. Eur J Nucl Med. 1999; 26: S36-42.
11. Mariani G, Moresco L, Viale G, Villa G, Bagnasco M, Canavese G, et al. Radioguided sentinel lymph node biopsy in breast cancer surgery. J Nucl Med. 2001; 42: 1198-215.

12. Mariani G, Gipponi M, Moresco L, Villa G, Bartolomei M, Mazzarol G, et al. Radioguided sentinel lymph node biopsy in malignant cutaneous melanoma. J Nucl Med. 2002; 43: 811-27.

13. Somasundaram SK, Chicken DW, Keshtgar MR. Detection of the sentinel lymph node in breast cancer. Br Med Bull. 2007; 84: 117-31.

14. Brouwer OR, van den Berg NS, Matheron HM, van der Poel HG, van Rhijn BW, Bex A, et al. A hybrid radioactive and fluorescent tracer for sentinel node biopsy in penile carcinoma as a potential replacement for blue dye. Eur Urol. 2014; 65: 600-9.

15. Subramanian S, Dandekar P, Jain R, Pandey U, Samuel G, Hassan PA, et al. Technetium-99m-labeled poly(DL-lactide-co-glycolide) nanoparticles as an alternative for sentinel lymph node imaging. Cancer Biother Radiopharm. 2010; 25: 637-44.

16. Schaafsma BE, Mieog JS, Hutteman M, van der Vorst JR, Kuppen PJ, Lowik $\mathrm{CW}$, et al. The clinical use of indocyanine green as a near-infrared fluorescent contrast agent for image-guided oncologic surgery. J Surg Oncol. 2011; 104: 323-32.

17. Niu G, Chen X. Lymphatic imaging: focus on imaging probes. Theranostics. 2015; 5: 686-97.

18. Niu G, Sun X, Cao Q, Courter D, Koong A, Le QT, et al. Cetuximab-based immunotherapy and radioimmunotherapy of head and neck squamous cell carcinoma. Clin Cancer Res. 2010; 16: 2095-105.

19. Herbst RS. Review of epidermal growth factor receptor biology. Int J Radiat Oncol Biol Phys. 2004; 59: 21-6.

20. Oda K, Matsuoka Y, Funahashi A, Kitano H. A comprehensive pathway map of epidermal growth factor receptor signaling. Mol Syst Biol. 2005; 1: 2005 0010 .

21. Rimawi MF, Shetty PB, Weiss HL, Schiff R, Osborne CK, Chamness GC, et al. Epidermal growth factor receptor expression in breast cancer association with biologic phenotype and clinical outcomes. Cancer. 2010; 116: 1234-42.

22. Sainsbury JR, Farndon JR, Needham GK, Malcolm AJ, Harris AL. Epidermal-growth-factor receptor status as predictor of early recurrence of and death from breast cancer. Lancet. 1987; 1: 1398-402.

23. Cai W, Niu G, Chen X. Multimodality imaging of the HER-kinase axis in cancer. Eur J Nucl Med Mol Imaging. 2008; 35: 186-208.

24. Jackson DG, Prevo R, Clasper S, Banerji S. LYVE-1, the lymphatic system and tumor lymphangiogenesis. Trends Immunol. 2001; 22: 317-21.

25. Guide for the care and use of laboratory animals. Washington, DC: National Academy Press. 2010.

26. Niu G, Zhu L, Ho DN, Zhang F, Gao H, Quan Q, et al. Longitudinal bioluminescence imaging of the dynamics of doxorubicin induced apoptosis. Theranostics. 2013; 3: 190-200.

27. Niu G, Lang L, Kiesewetter DO, Ma Y, Sun Z, Guo N, et al. In Vivo Labeling of Serum Albumin for PET. J Nucl Med. 2014; 55: 1150-6.

28. Banerji S, Ni J, Wang SX, Clasper S, Su J, Tammi R, et al. LYVE-1, a new homologue of the CD44 glycoprotein, is a lymph-specific receptor for hyaluronan. J Cell Biol. 1999; 144: 789-801.

29. Prevo R, Banerji S, Ferguson DJ, Clasper S, Jackson DG. Mouse LYVE-1 is an endocytic receptor for hyaluronan in lymphatic endothelium. J Biol Chem. 2001; 276: 19420-30.

30. Wang $Y$, Lang L, Huang $P$, Wang Z, Jacobson $O$, Kiesewetter DO, et al. In vivo albumin labeling and lymphatic imaging. Proc Natl Acad Sci U S A. 2015;112:208-13

31. Newman EA, Newman LA. Lymphatic mapping techniques and sentinel lymph node biopsy in breast cancer. Surg Clin North Am. 2007; 87: 353-64, viii.

32. Tausch C, Baege A, Rageth C. Mapping lymph nodes in cancer management role of (99m)Tc-tilmanocept injection. Onco Targets Ther. 2014; 7: 1151-8.

33. Huang X, Zhang F, Lee S, Swierczewska M, Kiesewetter DO, Lang L, et al. Long-term multimodal imaging of tumor draining sentinel lymph nodes using mesoporous silica-based nanoprobes. Biomaterials. 2012; 33: 4370-8.

34. Kim S, Lim YT, Soltesz EG, De Grand AM, Lee J, Nakayama A, et al. Near-infrared fluorescent type II quantum dots for sentinel lymph node mapping. Nat Biotechnol. 2004; 22: 93-7.

35. Verbeek FP, Troyan SL, Mieog JS, Liefers GJ, Moffitt LA, Rosenberg M, et al. Near-infrared fluorescence sentinel lymph node mapping in breast cancer: a multicenter experience. Breast Cancer Res Treat. 2014; 143: 333-42.

36. Feng X, Chen A, Zhang Y, Wang J, Shao L, Wei L. Central nervous system toxicity of metallic nanoparticles. Int J Nanomedicine. 2015; 10: 4321-40.

37. Harisinghani MG, Barentsz J, Hahn PF, Deserno WM, Tabatabaei S, van de $\mathrm{Kaa} \mathrm{CH}$, et al. Noninvasive detection of clinically occult lymph-node metastases in prostate cancer. N Engl J Med. 2003; 348: 2491-9.

38. Yang L, Saija HK, Cao Z, Qian W, Bender L, Marcus AI, et al. uPAR-targeted optical imaging contrasts as theranostic agents for tumor margin detection. Theranostics. 2013; 4: 106-18

39. Pribluda A, de la Cruz CC, Jackson EL. Intratumoral Heterogeneity: From Diversity Comes Resistance. Clin Cancer Res. 2015; 21: 2916-23.

40. Mishani E, Abourbeh G, Eiblmaier M, Anderson CJ. Imaging of EGFR and EGFR tyrosine kinase overexpression in tumors by nuclear medicine modalities. Curr Pharm Des. 2008; 14: 2983-98.

41. Maurer T, Eiber M, Schwaiger M, Gschwend JE. Current use of PSMA-PET in prostate cancer management. Nat Rev Urol. 2016; 13: 226-35. 
42. Sugiyama K, Kawai T, Nagata N, Suzuki M. Tumor-associated carbohydrate antigens in primary pulmonary adenocarcinomas and their metastases. Hum Pathol. 1992; 23: 900-4.

43. Sainte-Marie G, Peng FS. Structural and cell population changes in the lymph nodes of the athymic nude mouse. Lab Invest. 1983; 49: 420-9.

44. Economopoulos V, Noad JC, Krishnamoorthy S, Rutt BK, Foster PJ. Comparing the MRI appearance of the lymph nodes and spleen in wild-type and immuno-deficient mouse strains. PLoS One. 2011; 6: e27508. 